PROCEEDINGS OF THE

AMERICAN MATHEMATICAL SOCIETY

Volume 133, Number 11, Pages 3355-3360

S 0002-9939(05)07922-0

Article electronically published on May 9, 2005

\title{
ON SUCCESSIVE COEFFICIENTS OF ODD UNIVALENT FUNCTIONS
}

\author{
ZHONGQIU YE
}

(Communicated by Juha M. Heinonen)

\begin{abstract}
The relative growth of successive coefficients of odd univalent functions is investigated. We prove that a conjecture of Hayman is true.
\end{abstract}

\section{INTRODUCTION}

Let $S$ be the class of functions $f(z)=z+a_{2} z^{2}+\ldots$ regular and univalent in $|z|<1$ and let $S_{2}$ be the subclass of odd functions $f(z)=\sum_{k=0}^{\infty} b_{k} z^{2 k+1}$ in $S$. In the investigation of the relative growth of successive coefficients of functions in $S_{2}$, Goluzin [1] has established the inequality

$$
|| b_{n}|-| b_{n-1}|| \leq A n^{-1 / 4} \ln n(n=2,3, \ldots),
$$

where $A$ is an absolute constant. In 1963, W. Hayman [2] formulated the conjecture that

$$
|| b_{n}|-| b_{n-1}|| \leq A(\varepsilon) n^{-1 / 2+\varepsilon}
$$

for every $\varepsilon>0$. Lucas [3] and Huke [4], coming close to this conjecture, proved respectively the estimates

$$
|| b_{n}|-| b_{n-1}||=O\left(n^{-b}\right),
$$

where $b=\sqrt{2}-1$ and $b=0.42667$. In this paper we shall prove Hayman's conjecture. If $f_{2} \in S_{2}, f_{2}(z)=\sqrt{ } f\left(z^{2}\right)=\sum_{k=0}^{\infty} b_{k} z^{2 k+1}$, where $f$ is in $S$. Thus we only need to study the successive coefficients of the function $(f(z) / z)^{\frac{1}{2}}=\sum_{k=0}^{\infty} b_{k} z^{k}$, where $f$ is in $S$. Our main result is

Theorem 1.1. Let $f \in S$ and let the coefficients $b_{k}(k=0,1,2, \ldots)$ be defined by the expansion

$$
(f(z) / z)^{1 / 2}=\sum_{k=0}^{\infty} b_{k} z^{k}
$$

Then for $n=2,3, \ldots$,

$$
|| b_{n}|-| b_{n-1}|| \leq A n^{-1 / 2} \log n,
$$

where $A$ is an absolute constant.

Received by the editors August 23, 2002 and, in revised form, June 24, 2004.

2000 Mathematics Subject Classification. Primary 30C45.

Key words and phrases. Successive coefficients, univalent functions.

(C)2005 American Mathematical Society Reverts to public domain 28 years from publication 
The exponent $-\frac{1}{2}$ is sharp in Theorem 1.1, since

$$
f(z)=z\left(1-z^{4}\right)^{-\frac{1}{2}}=\sum_{k=0}^{\infty} b_{2 k+1} z^{2 k+1}
$$

is univalent in $|z|<1$ and

$$
b_{4 k+1} \sim k^{-\frac{1}{2}} / \sqrt{ } \pi, k \rightarrow \infty,
$$

while

$$
b_{4 k-1}=0 .
$$

\section{AN AUXILIARY RESUlT}

Assume, as usual, that the logarithmic coefficients $2 \gamma_{k}(k=1,2, \ldots)$ of the function $f(z) \in S$ are defined by the expansion

$$
\log (f(z) / z)=2 \sum_{k=1}^{\infty} \gamma_{k} z^{k} .
$$

Theorem 2.1. Let $f \in S$. Let $r_{1}=(1-1 / n)^{\frac{1}{2}}, r_{2}=(1-1 / 2 n)^{\frac{1}{2}}(n=2,3, \ldots)$. If $t$ is chosen such that $|f(t)|=\max \left\{|f(z)|:|z|=r_{2}\right\}$ and if $z=r_{1} e^{i \theta}$, then

$$
\int_{0}^{2 \pi}\left|\frac{f(z)}{z}\left(1-t^{-1} z\right)\right| d \theta \leq A \log n,
$$

where $A$ is an absolute constant.

Proof. We consider the function $g(z)=1 / f\left(z^{-1}\right)(|z|>1)$ in $\sum$ which satisfies

$$
1 / g(z)=f\left(z^{-1}\right)=z^{-1}+\sum_{n=2}^{\infty} a_{n} z^{-n} .
$$

Let $b_{k l}(k, l=1,2, \ldots)$ be the Grunsky coefficients of $g(z)$. We define

$$
\begin{gathered}
\alpha_{k}=\alpha_{k}(s)=\sum_{l=1}^{\infty} b_{k l} s^{-l}(|s|>1, k=1,2, \ldots), \\
h=h(z, s)=\frac{z-s}{g(z)-g(s)}=\sum_{\nu=0}^{\infty} \beta_{\nu}(s) z^{-\nu} \quad(|z|,|s|>1) .
\end{gathered}
$$

It follows that for a fixed $s$ (see [7, p. 82),

$$
\frac{d}{d z} \log h=\frac{1}{z-s}-\frac{g^{\prime}(z)}{g(z)-g(s)}=-\sum_{k=0}^{\infty} k \alpha_{k}(s) z^{-k-1}
$$

and

$$
\frac{d}{d z} h=\frac{1}{g(z)-g(s)}-\frac{(z-s) g^{\prime}(z)}{(g(z)-g(s))^{2}}=-\sum_{\nu}^{\infty} \nu \beta_{\nu}(s) z^{-\nu-1} .
$$

The Grunsky inequality shows that (see [7], p. 83)

$$
\sum_{k=1}^{\infty} k\left|\alpha_{k}(r)\right|^{2} \leq \sum_{k=1}^{\infty} \frac{r^{-2 k}}{k}=\log \frac{1}{1-r^{-2}} .
$$


Write $\beta_{\nu}=\beta_{\nu}(s)$. Let $|s|=\rho=r_{2}^{-1}$. Applying Milin's theorem (see [7], p. 80), we obtain from (12) that for $n=1,2, \ldots$,

$$
\left|\beta_{n}(r)\right|^{2} \leq \exp \left\{\sum_{k=1}^{n} k\left|\alpha_{k}\right|^{2}\right\} \leq n^{-1} \exp \left\{\sum_{k=1}^{\infty} k\left|\alpha_{k}\right|^{2}\right\} \leq n^{-1} \frac{1}{1-\rho^{-2}} \leq A,
$$

where $A$ is an absolute constant. We obtain from (9), (10) and (11) that (see [7, p. 82)

$$
\frac{d}{d z} \frac{z-s}{g(z)}=\left(1-\frac{g(s)}{g(z)}\right) h \frac{d}{d z} \log h-g(s) h \frac{d}{d z} \frac{1}{g(z)} .
$$

We choose a fixed $s$ such that

$$
|g(s)|=\min \{|g(z)|:|z|=\rho\}=\frac{1}{M\left(\frac{1}{\rho}\right)}
$$

where

$$
M\left(\frac{1}{\rho}\right)=\max \left\{|f(z)|:|z|=\frac{1}{\rho}\right\} .
$$

Write $z=r e^{i \theta}, r \in[\rho, 2], \theta \in[0,2 \pi]$ and $\lambda=r_{1}^{-1}(n=1,2, \ldots)$. We obtain from (14) that

$$
\begin{gathered}
\int_{0}^{2 \pi} \int_{\lambda}^{2}\left|\frac{d}{d z} \frac{z-s}{g(z)}\right| d r d \theta \\
\leq \int_{0}^{2 \pi} \int_{\lambda}^{2}\left|1-\frac{g(s)}{g(z)}\right|\left|h \frac{d}{d z} \log h\right| d r d \theta+\int_{0}^{2 \pi} \int_{\lambda}^{2}|g(s)|\left|h \frac{d}{d z} \frac{1}{g(z)}\right| d r d \theta .
\end{gathered}
$$

Now we estimate the two terms $I_{1}$ and $I_{2}$ on the right-hand side of (17).

(a) Since $|g(s)| \leq|g(z)|$, by (15) and (16), we obtain from Schwarz's inequality and (9) and (10) that

$$
\begin{aligned}
\left|I_{1}\right| & \leq 2\left[\int_{0}^{2 \pi} \int_{\lambda}^{2}|h|^{2} d \theta d r\right]^{\frac{1}{2}}\left[\int_{0}^{2 \pi} \int_{\lambda}^{2}\left|\frac{d}{d z} \log h\right|^{2} d \theta d r\right]^{\frac{1}{2}} \\
& \leq 4 \pi\left[\int_{\lambda}^{2} \sum_{\nu=0}^{\infty}\left|\beta_{\nu}\right|^{2} r_{-2 \nu} d r\right]^{\frac{1}{2}}\left[\int_{\lambda}^{2} \sum_{\nu=1}^{\infty} \nu^{2}\left|\alpha_{\nu}\right|^{2} r^{-2 \nu-2}\right]^{\frac{1}{2}} \\
& \leq 4 \pi\left[2+\sum_{\nu=1}^{\infty} \frac{1}{2 \nu-1}\left|\beta_{\nu}\right|^{2} \lambda^{-2 \nu+1}\right]^{\frac{1}{2}}\left[\sum_{\nu=1}^{\infty} \frac{\nu}{2 \nu+1} \nu\left|\alpha_{\nu}\right|^{2} \lambda^{-2 \nu-1}\right]^{\frac{1}{2}} .
\end{aligned}
$$

It follows from (12), (13) and (18) that

$$
\left|I_{1}\right| \leq A \log \frac{1}{1-\lambda^{-2}} \leq A \log n
$$

where $A$ is an absolute constant. 
(b) We apply Schwarz's inequality again to estimate $I_{2}$. It follows from (7) and (13) that

$$
\begin{aligned}
\left|I_{2}\right| & \leq|g(s)|\left[\int_{\lambda}^{2} \int_{0}^{2 \pi}|h|^{2} d \theta d r\right]^{\frac{1}{2}}\left[\int_{\lambda}^{2} \int_{0}^{2 \pi}\left|\frac{d}{d z} \frac{1}{g(z)}\right|^{2} d \theta d r\right]^{\frac{1}{2}} \\
& \leq 2 \pi|g(s)|\left[2+2+\sum_{\nu=1}^{\infty} \frac{1}{2 \nu-1}\left|\beta_{\nu}\right|^{2} \lambda^{-2 \nu+1}\right]^{\frac{1}{2}}\left[\int_{\lambda}^{2} \sum_{\nu=1}^{\infty} \nu^{2}\left|\alpha_{\nu}\right|^{2} r^{-2 \nu} d r\right]^{\frac{1}{2}} \\
& \leq A|g(s)|\left[\log \frac{1}{1-\rho^{-2}}\right]^{\frac{1}{2}}\left[\sum_{\nu=1}^{\infty} \frac{\nu}{2 \nu-1} \nu\left|\alpha_{\nu}\right|^{2} \lambda^{-2 \nu+1}\right]^{\frac{1}{2}},
\end{aligned}
$$

where $A$ is an absolute constant. Because $f(z)$ is univalent in $|z|<1$, we obtain from (15) and (16) that

$$
\sum_{\nu=1}^{\infty} \frac{\nu}{2 \nu-1} \nu\left|\alpha_{\nu}\right|^{2} \lambda^{-2 \nu} \leq \frac{1}{\pi} \iint_{|z|<1 / \rho}\left|f^{\prime}(z)\right|^{2} d \sigma \leq\left(M\left(\frac{1}{\rho}\right)\right)^{2}=|g(s)|^{2} .
$$

We obtain from (20) and (21) that

$$
\left|I_{2}\right| \leq A \log \frac{1}{1-\rho^{-2}} \leq A \log n,
$$

where $A$ is an absolute constant. The estimate obtained in (a) and (b) for the terms on the right-hand side of (17) shows that there exists an absolute constant $A$ such that

$$
\int_{0}^{2 \pi} \int_{\lambda}^{2}\left|\frac{d}{d z} \frac{z-s}{g(z)}\right| d r d \theta \leq A \log n
$$

Let $z_{1}=2 e^{i \theta}, z_{2}=\lambda e^{i \theta}$ and $z=r e^{i \theta}, r \in[\rho, 2]$. We obtain from (23) that

$$
\begin{gathered}
\int_{0}^{2 \pi}\left|\frac{z_{2}-s}{g\left(z_{2}\right)}\right| d \theta-\int_{0}^{2 \pi}\left|\frac{z_{1}-s}{g\left(z_{1}\right)}\right| d \theta \leq \int_{0}^{2 \pi} \int_{\lambda}^{2}\left|\frac{\partial}{\partial r}\right| \frac{z-s}{g(z)}|| d r d \theta \\
\leq \int_{0}^{2 \pi} \int_{\lambda}^{2}\left|\frac{d}{d z} \frac{z-s}{g(z)}\right| d r d \theta \leq A \log n .
\end{gathered}
$$

It is clear that

$$
\int_{0}^{2 \pi}\left|\frac{z_{1}-s}{g\left(z_{1}\right)}\right| d \theta \leq 2 \pi M\left(\frac{1}{2}\right)\left(\left|z_{1}\right|+|s|\right) \leq A,
$$

where $A$ is an absolute constant. We obtain from (24) and (25) that

$$
\int_{0}^{2 \pi}\left|f\left(z_{2}^{-1}\right)\right|\left|z_{2}^{-1}-s^{-1}\right| d \theta=\frac{1}{\left|z_{2} s\right|} \int_{0}^{2 \pi}\left|\frac{z_{2}-s}{g\left(z_{2}\right)}\right| d \theta \leq A \log n,
$$

where $A$ is an absolute constant. The inequality (26) implies for $z=r_{1} e^{i \theta},|t|=r_{2}$ and $|f(t)|=\max \left\{|f(z)|:|z|=r_{2}\right\}$ that

$$
\int_{0}^{2 \pi}|f(z)||z-t| d \theta \leq A \log n
$$




\section{Proof of Theorem 1.1}

Proof. We define two functions and give their power series expansions (we choose single-valued brunch with value 1 at $z=0$ ) when $|z|<r_{2}$ :

$$
\begin{gathered}
u(z)=(f(z) / z)^{1 / 2}\left(1-t^{-1} z\right)^{1 / 2}=\sum_{k=0}^{\infty} d_{k} z^{k} \\
v(z)=u(z)\left(1-t^{-1} z\right)^{1 / 2}=(f(z) / z)^{1 / 2}\left(1-t^{-1} z\right)=\sum_{k=0}^{\infty} c_{k} z^{k},
\end{gathered}
$$

where $|t|=r_{2}=[1-1 /(2 n)]^{\frac{1}{2}}$ and $|f(t)|=\max \left\{|f(z)|:|z|=r_{2}\right\}$. Theorem 2.1 shows that

$$
\sum_{k=0}^{\infty}\left|d_{k}\right|^{2} r_{1}^{2 k}=\frac{1}{2 \pi} \int_{0}^{2 \pi}\left|u\left(r_{1} e^{i \theta}\right)\right|^{2} d \theta \leq A \log n
$$

Hence, we obtain from (30) that

$$
\sum_{k=0}^{\infty}\left|c_{k}\right|^{2} r_{1}^{2 k}=\frac{1}{2 \pi} \int_{0}^{2 \pi}\left|v\left(r_{1} e^{i \theta}\right)\right|^{2} d \theta \leq \frac{2}{2 \pi} \int_{0}^{2 \pi}\left|u\left(r_{1} e^{i \theta}\right)\right|^{2} d \theta \leq A \log n,
$$

where $A$ is an absolute constant. Let

$$
\log v(z)=\sum_{k=1}^{\infty} A_{k} z^{k}
$$

for $|z|<r_{2}$. Then it follows from (29) that

$$
A_{k}=\gamma_{k}-t^{-k} / k \quad(k=1,2, \ldots) .
$$

It is clear that $v^{\prime}(z)=v(z)[\log v(z)]^{\prime}$. Comparing coefficients, we obtain the recursion formula

$$
n c_{n}=\sum_{k=1}^{n} k A_{k} c_{n-k} .
$$

Applying Schwarz's inequality to the recursion formula (34), we obtain that

$$
\begin{gathered}
n^{2}\left|c_{n}\right|^{2} r_{1}^{2 n} \leq \sum_{k=1}^{n} k^{2}\left|A_{k}\right|^{2} r_{1}^{2 k} \sum_{k=0}^{n}\left|c_{k}\right|^{2} r_{1}^{2 k} \leq n \sum_{k=1}^{n} k\left|A_{k}\right|^{2} r_{1}^{2 k} \sum_{k=0}^{n}\left|c_{k}\right|^{2} r_{1}^{2 k} \\
\leq n \sum_{k=1}^{\infty} k\left|A_{k}\right|^{2} r_{1}^{2 k} \sum_{k=0}^{\infty}\left|c_{k}\right|^{2} r_{1}^{2 k} .
\end{gathered}
$$

It follows by (33) that

$$
\sum_{k=1}^{\infty} k\left|A_{k}\right|^{2} r_{1}^{2 k} \leq 2\left(\sum_{k=1}^{\infty} k\left|\gamma_{k}\right|^{2} r_{1}^{2 k}+\sum_{k=1}^{\infty} \frac{1}{k}\left(\frac{r_{1}}{r_{2}}\right)^{2 k}\right)
$$

Since $r_{1} / r_{2}<r_{2}$, it follows that

$$
\sum_{k=1}^{\infty} k^{-1}\left(\frac{r_{1}}{r_{2}}\right)^{2 k}<\sum_{k=1}^{\infty} k^{-1} r_{2}^{2 k}=\log \frac{1}{1-r_{2}^{2}}<\log 2 n .
$$


On the other hand, we have (see [6], pp. 283-290)

$$
\sum_{k=1}^{\infty} k\left|\gamma_{k}\right|^{2} r_{1}^{2 k} \leq \max \left\{\log |f(z) / z|:|z|=r_{1}\right\} \leq \log \frac{1}{\left(1-r_{1}\right)^{2}}<2 \log n .
$$

Combining (37) and (38), we obtain from (36) that

$$
\sum_{k=1}^{\infty} k\left|A_{k}\right|^{2} r_{1}^{2 k} \leq 6 \log 2 n
$$

Thus, it follows from (31), (39) and (35) that

$$
\left|c_{n}\right| \leq A n^{-1 / 2} \log n
$$

where $A$ is an absolute constant. It follows from (29) that $c_{n}=b_{n}-t^{-1} b_{n-1}$. Hence, we have

$$
|| b_{n}|-| b_{n-1}|| \leq\left|c_{n}\right|+\left(\left[1-\frac{1}{2 n}\right]^{-\frac{1}{2}}-1\right)\left|b_{n-1}\right| .
$$

It is well known that $\left|b_{n}\right|<1.17(n=1,2, \ldots)$ (see [5]). We obtain from (40) and (41) that

$$
|| b_{n}|-| b_{n-1}|| \leq A n^{-\frac{1}{2}} \log n+O\left(\frac{1}{n}\right) .
$$

Finally, Theorem 1 follows from (42).

\section{REFERENCES}

[1] G.M. Goluzin, Geometric Theory of Functions of a Complex Variable, Moscow, 1952.

[2] W.K. Hayman, On successive Coefficients of Univalent Functions, J. London. Math. Soc. 38 (1963), 228-243. MR0148885|(26:6382)

[3] K.W. Lucas, On Successive Coefficients of Areally Mean-p valent Functions, J. London. Math. Soc, 44 (1969), 631-642. MR0243055 (39:4379)

[4] Hu Ke, On Succcessive Coefficients of Univalent Functions, Proc. Amer. Math. Soc. 95 (1986), 37-41. MR0796442 (86j:30022)

[5] I.M. Milin, Univalent Functions and Orthonomal System (Russian) Moscow, 1971.

[6] V.I. Milin, Adjacent Coefficients of Odd Univalent Functions, Sib. Math. Journal 22 (1981), no. 2, 283-290. MR0610775 (82i:30027)

[7] Ch. Pommerenke, Univalent Functions, Vandenhoeck \& Ruprecht, Göttingen, 1975. MR0507768 (58:22526)

Department of Mathematics, Jiangxi Normal University, Nanchang 330027, People's Republic of China

E-mail address: yezhqi@sina.com 